\title{
Moon in-situ resources: clues from the study of Lunar achondrites in preparation for Artemis sample return missions
}

\author{
Josep Maria Trigo-Rodríguez ${ }^{1,2}$, Esther Mas-Sanz ${ }^{2,3}$, Jordi Ibáñez-Insa ${ }^{4}$, and Jacinto Alonso- \\ Azcárate ${ }^{5}$ \\ ${ }^{1}$ Institute of Space Sciences (ICE, CSIC), Meteorites, Minor Bodies and Planetary Science Group, Campus UAB, Carrer de Can \\ Magrans, s/n, 08193 Cerdanyola del Vallés, Barcelona, Catalonia, Spain (trigo@ice.csic.es) \\ ${ }^{2}$ Institut d'Estudis Espacials de Catalunya (IEEC), Gran Capità 2-4, Ed. Nexus, desp. 201, 08034 Barcelona, Catalonia, Spain \\ ${ }^{3}$ ESEIAAT, Universitat Politècnica de Catalunya (UPC), 08222 Terrassa, Catalonia, Spain \\ ${ }^{4}$ Institute of Earth Sciences Jaume Almera (ICTJA-CSIC), C/Lluís Solé i Sabarís, s/n, 08028 Barcelona, Catalonia, Spain; \\ ${ }^{5}$ Fac. CC Ambientales y Bioquímica, Universidad de Castilla-La Mancha, Avda. Carlos III s/n, 45071 Toledo, Spain
}

\section{Introduction.}

The Moon is no longer a goal in itself but a necessary step for the conquest of space. In this work we focus in the composition of Lunar meteorites as the only objects alongside Apollo, and Luna collected samples, that the scientific community has available to first-hand analyze the Moon. Future Artemis sample return missions will provide new samples to continue learning from our satellite. Given its proximity to our planet, our satellite is an ideal planetary body to use it as a space camp for new technology, to establish a space base, and to test space mining. The continuous depletion of Earth resources put special importance on the exploration of extraterrestrial natural resources potential and the feasibility of its exploitation. After many robotic and manned missions, the utilization of lunar resources has been studied for a long time. In-Situ Resources Utilization (ISRU) refers to the generation of materials (for construction, life support, or as propellants) from the available resources on a celestial body that otherwise, would have needed to be brought from Earth [1].

\section{Analitical Techniques and Samples}

For the present work, four different Lunar achondrites (Lunaites) were studied: Dhofar 1084, Jiddat al Harasis 838, Northwest Africa 2700 and 11444, and Miller Range 090031 (abbreviated in Table 1 ). Thin sections of each meteorite allow us a characterization of the mineralogy using SEM/EDX and optical microscopy (Fig. 1). In addition, XRD measurements on capillary powder samples of the meteorites are made using a powder diffractomer equipped with a Mo X-ray souce $(\lambda=0.709 \AA)$. This experimental configuration allows us to minimize preferential orientation effects as well as to significantly reduce the X-ray fluorescence signal from Fe, relative to XRD measurements performed with a standard $\mathrm{Cu}$ X-ray tube. Finally we perform ICP-MS and ICP-AES of the samples using a similar procedure that in our previous studies of meteorites [2]. Meteorite specimens studied so far are listed in Table 1. 


\begin{tabular}{|c|c|c|c|}
\hline Meteorite & Type & Prob. origin & TKM (g) \\
\hline Dho 1084 & Lunar (anorthositic) & Lunar mare & 90 \\
\hline JaH 838 & Lunar (mingled regolith breccia) & Lunar highlands & 34.4 \\
\hline NWA 2700 & olivine gabbro with regolith breccia & & 31.7 \\
\hline NWA 11444 & Lunar (melt breccia) & Lunar highlands & 1323 \\
\hline
\end{tabular}

Table 1. Lunar meteorites under study, type, probable origin and Total Known Mass (TKM).

\section{Discussion}

Like the Lunar rocks, Lunaites provide valuable information about the chemical and mineralogical complexity that can be found in the surface of the Moon. Despite that we don't know exactly their origin in the Lunar surface, these relatively small rocks provide clues on the processes going on in the surface of the Moon. Among the most fascinating meteorites, JaH 838 is a mingled regolith breccia presenting mare and KREEPy clasts, together with the products of thermal processing: HighAl Si-Poor (HASP) glasses with chondritic metal grains. Another one is the complex melt breccia NWA 11444 containing a wide variety of angular fragments (gabbros and basalts) and variable amounts of flow-banded glass. Crystal fragments consist predominantly of plagioclase, pyroxene, and olivine. Finally the rock also contains a few percent of $\mathrm{Fe}, \mathrm{Ni}$ metal grains probably reminiscence of the projectiles sculpting the rock (see Fig. 1).

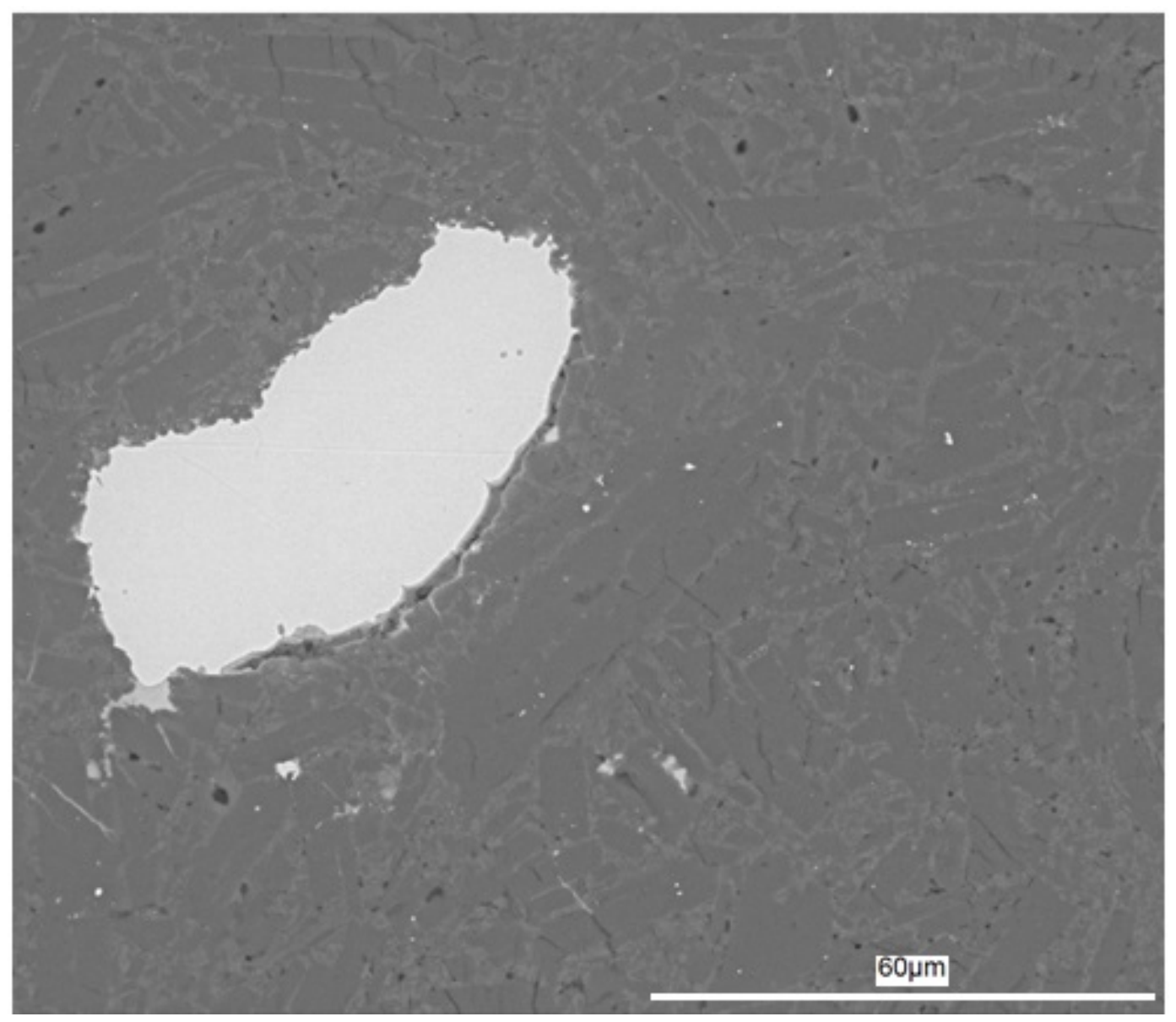

Fig. 1. A kamacite grain set into an anorthite-rich matrix of NWA 11444.

\section{Conclusions.}

From our study and having into account our current knowledge of the chemical and mineralogical 
lunar resources which can be realistically used for ISRU, the following resources are considered $[3,4]$ :

- Metals hosted in the lunar regolith are mostly due to the continuous impact of chondritic and metallic projectiles with the Lunar surface. As a consequence, the regolith is enriched in minerals such as pyroxene, olivine, ilmenite and native metals such as $\mathrm{Fe}$ and $\mathrm{Ni}$. In addition, it can be found hydrated minerals and rare-Earth elements. As most metals are found in the form of oxides (as well as some pure kamacite), it makes their extraction costly energy-wise as these components tend to be chemically stable [3], but their potential use for producing spacecraft parts and in-situ repairs make them more attractive. On the other hand, the extraction of oxygen is especially interesting from a biological point of view: future missions may utilize it for the production of water and other life support processes.

- Water, probably to be found as ice inside permanently shadowed craters of the polar regions, could be used as rocket fuel and to support life in a Moon base. On the other hand, some regions of the Moon have been hit by carbonaceous chondritic asteroids, rich in clay minerals. These hydrous minerals contain absorbed and bound water. If heated at temperatures $\square 100-150{ }^{\circ} \mathrm{C}$ absorbed water can be released and bound water at $\square 300{ }^{\circ} \mathrm{C}[4]$.

- Carbon and other organic compounds are also common in regolith-rich regions of the Moon. There is a dominant flux of CM chondrites [5] that after impact dehydrate and end up as "graphitized" clasts observed in regolith breccias [6]. Furthermore, it has been theorized of an uncertain amount of hydrocarbons could be used for the production of much complex polymers, resins and plastics [1].

- Finally, solar wind volatiles become also implanted in the Lunar regolith: $\mathrm{H}, \mathrm{N}, \mathrm{C}$ and in particular the $\mathrm{He}-3$ isotope, rare to find on Earth and key for future developments in nuclear fusion.

\section{Conclusions}

Lunar meteorites are valuable samples teaching us about the processes going on over the Moon, at the same time that provide clues on the most important minerals for mining. Many different initiatives on how to tackle Lunar resources are taking place: from 3-D printers that previously construct the necessary infrastructure for lunar mining, the utilization of autonomous robots and obviously, the mingle of different proposals according to the exploitation stage. The Lunar surface provides a lot of valuable materials, but a precise know-how is required to successfully exploit them. Then, a careful study of Lunar samples and meteorites will provide significant progress in optimizing ISRU.

\section{Acknowledgements}

The authors acknowledge financial support from the Spanish Ministry (PGC2018-097374-B-I00).

\section{References}

- ISRU NASA Homepage: https://isru.nasa.gov/

- Herrero, M. J.; Trigo-Rodríguez, J. M.; Alonso-Azcárate, J., 2019, 50thLPSC. LPI Contribution No. 2132, id.1839.

- Anand M. et al., 2012, Planet. Space Sci., 74(1), 42, doi: 10.1016/j.pss.2012.08.012.

- Trigo-Rodríguez J. M. et al.,2019, Space Sci. Rev., 215(1), 18, doi: 10.1007/s11214-019-0583-0.

- Warren P.H. and Taylor G.J.,2014, In Treatise in Cosmochemistry, $2^{\text {nd }}$ ed., Elsevier Ltd., doi: 10.1016/B978-0-08-095975-7.00124-8

- Rubin A.E. et al.,2014, Geochim. et Cosmoch. Acta 69, 3419, https://doi.org/10.1016/j.gca.2004.11.001 